\title{
A Convenient Method for Catalytic Aromatic Pentafluoroethylation Using Potassium (Pentafluoroethyl)trimethoxyborate
}

Tsuyuka Sugiishi

Daisuke Kawauchi

Mizuki Sato

Tatsuya Sakai

Hideki Amii*

Synthesis 2017, 49, 1874.

In the general procedure for the synthesis of potassium (pentafluoroethyl)trimethoxyborate, 'trifluoromethoxyborate' was replaced by 'trimethyl borate'. 